\title{
A DIPLOMACIA CULTURAL NO GOVERNO LULA (2003-20II)
}

\author{
THE CULTURAL DIPLOMACY IN THE LULA GOVERNMENT (2003-20II)
}

\author{
Osvaldo Alencar Billig1 \\ Rafaela Maria Souza Farias ${ }^{2}$
}

RESUMO: Pós a onda de globalização no mundo, a diplomacia cultural se tornou uma ferramenta de extremo interesse para a política externa de atores internacionais. A ferramenta consiste no uso da cultura como objeto para construção da imagem de ator âmbito internacional, tendo como função gerar aproximação cultural, redução das desconfianças, fomentar as relações entre atores de forma a beneficiar vínculos políticoeconômicos. Este artigo objetiva analisar a diplomacia cultura no período do Governo Lula, buscando entender seus propósitos e estratégias. A metodologia utilizada se dá através da revisão bibliográfica de literatura especializada e analise documental pautada em trabalhos acadêmicos e livros. O resultado encontrado foi que a diplomacia cultura do Governo Lula buscou construir a imagem de um Brasil mediador de conflitos, buscando sempre a via do diálogo. Por fim, instiga-se a reflexão sobre o funcionamento da ferramenta citada em um país vasto com muitas culturas como o Brasil.

Palavras-chave: cultura. Diplomacia cultural. Brasil.

ABSTRACT: After the wave of globalization in the world, cultural diplomacy has become a tool of extreme interest for the foreign policy of international actors. The tool consists in the use of culture as an object to build the image of an actor at an international level, with the function of generating cultural approximation, reducing distrust, and fostering relationships between actors in order to benefit political-economic ties. This article aims to analyze cultural diplomacy during the Lula government, seeking to understand its purposes and strategies. The methodology used is through the bibliographic review of specialized literature and documentary analysis based on academic works and books. The result found

\footnotetext{
I Bacharel em Administração pela Universidade de Passo Fundo (UPF); MBA em Logística Empresarial pela Fundação Getúlio Vargas (FGV); Mestre em Administração pela Universidade de Caxias do Sul (UCS). Email: probillig@gmail.com, ORCID: https://orcid.org/oooo-ooo2-4166-9232. Professor do Centro Universitário Dinâmica das Cataratas (UDC), onde ministro aulas nos cursos de Administração, Engenharia de Produção, Comunicação e especialmente no curso de Relações Internacionais, as disciplinas de Introdução à Economia, Diplomacia Corporativa, Projeto Integrador II e Teoria das Negociações. E- mail: probillig@gmail.com.

2 Acadêmica do Curso de Relações Internacionais do Centro Universitário Dinâmica das Cataratas (UDC Monjolo), Rua David Muffato, 367, Parque Monjolo, Foz do Iguaçu/PR. CEP 85.864-39o. ORCID: https://orcid.org/oooo-ooor-6632-889. E-mail: rafaelamsfarias@hotmail.com.
} 
was that the Lula government's cultural diplomacy sought to build the image of a conflict mediator Brazil, always seeking the way of dialogue. Finally, reflection on the functioning of the tool mentioned in a vast country with many cultures such as Brazil is instigated.

Keywords: culture, cultural diplomacy, Brazil.

\section{INTRODUÇÃO}

Ao se falar de diplomacia cultural, antes é necessário conceituar a palavra cultura, para assim atender aos fins corretos deste artigo. Nesse caso, será usado no sentido mais amplo dentro da antropologia, portanto, a cultura é a manifestação da identidade de um povo (indivíduos de um Estado) através de tradições, hábitos, língua e processo histórico.

Agora, para entender o que é a diplomacia cultural, entende-se a cultura como instrumento usado para promoção cultural de um Estado, e também para fins de interesses políticos, econômicos e sociais. Ou seja, a diplomacia cultural é, através da cultura, uma ferramenta estatal com o objetivo de produzir uma boa imagem de seu país, afim de facilitar a comunicação com outros, e também reduzir as inseguranças, para assim tornar processos econômicos e políticos mais passíveis de serem acordados. A diplomacia cultural, por sua vez, seria a utilização específica da relação cultural para a consecução de objetivos nacionais de natureza não somente cultural, mas também política, comercial e econômica (RIBEIRO, 2011, p.33).

Importante frisar que esse tipo de diplomacia tem interesse sim em estabelecer vínculos culturais entre Estados juntamente com seus indivíduos, que não fica somente a cargo dos interesses nacionais vinculados a política e economia. Outro ponto importante necessário de ser dito é sobre a quase inseparabilidade da sociedade com as questões políticas e econômicas, é intrínseco o movimento econômico e político na formação da sociedade e na participação de seus hábitos. Então é previsível, também, situações econômicas e políticas nas relações culturais estimuladas pela ferramenta de diplomacia.

No Brasil a diplomacia cultural tomou forma apenas em 1937 na Era Vargas, mas devido a seu período inicial sofreu muito com a organização institucional, muitos órgãos foram criados e ficaram muito dispersos, assim era dificultoso a definição das funções de cada um, e nos anos seguintes também teve muitas fusões, divisões e exclusões dos mesmos.

$\mathrm{Na}$ década de 1960, mais especificamente na ditadura militar, que a diplomacia cultural brasileira se tornou estável, contínua e com um propósito definido. Porém foramse longos anos até a diplomacia cultural ser percebida como um instrumento realmente útil e relevante para o Estado Brasileiro.

Nesse artigo, objetiva-se, então, realizar uma análise da diplomacia cultural durante o Governo Lula, e assim traçar quais eram seus propósitos, estratégias e contexto a qual se passava. Portanto, o artigo irá se subdividir em dois capítulos, primeiramente no Propósitos e objetivos, que visa contextualizar o cenário internacional onde o Brasil estava inserido e metas que o Governo Lula pretendia, segundamente em Meios e estratégias, com o intuito 
de mostrar e esclarecer as formas que o governo usou a diplomacia cultural para promoção do Estado. Por fim as considerações finais, que caberão a deixar reflexões para o leitor acerca das muitas culturas presentes no Brasil e o processo de escolha de qual será usada na diplomacia já citada, se é que há de ser feita uma escolha.

A metodologia usada neste artigo se caracteriza como pesquisa documental partindo de fontes secundárias, realizada através de revisão bibliográfica de publicações cientificas, como teses, artigos, e livros e trabalhos de autores como Amado Luiz Cervo, Clodoaldo Bueno e Edgard Ribeiro.

O motivo dessa pesquisa se define em termos de interesse na questão da relação cultura-diplomacia, por juntos se constituírem uma ferramenta que atende a interesses de um ator de forma branda, e envolver aspectos internos dos mesmos que vão se projetar externamente no meio internacional. Outro motivo também é o de contribuição para pesquisa brasileira sobre o assunto escolhido, pois, o meio acadêmico das Relações Internacionais sofre muita influência das pesquisas produzidos no centro do sistema internacional - como Estados Unidos, França e Inglaterra, assim pesquisas nas periferias, como o Brasil, ainda assumem um lugar pequeno no âmbito acadêmico e necessitam serem instigadas e realizadas em suas regiões de origem e posteriormente serem objetivadas ao resto do globo.

\section{FUNDAMENTAÇÃO TEÓRICA}

\section{I.I PROPÓSITOS E OBJETIVOS}

Antes de definir metas da diplomacia cultural no Governo Lula, é importante estabelecer características desse governo e também de entender que a diplomacia citada, mesmo tendo seus objetivos, é subordinada aos propósitos da política externa do Estado, isto é, atua em função de realizar objetivos próprios e da política externa, assim dando suporte ao conjunto inteiro dos propósitos traçados.

Caracterizando de maneira bem superficial o governo o qual será de estudos neste artigo, pode-se dizer que se desvinculava, de forma não profunda, do governo neoliberal anterior, que estava nas mãos de Fernando Henrique Cardoso, e procurava atender demandas ligadas mais ao meio social das necessidades da população, não que a economia não tivesse um papel importante, ainda sim era necessária e fundamental, mas o foco girava entorno da diminuição da pobreza, aumento do nível de escolaridade, o problema da fome e miséria, no geral, o foco era em melhorar as condições sociais do Brasil, e no Governo Lula a política externa foi usada como uma ferramenta para alcançar o desenvolvimento nacional, o que causaria simultaneamente a melhoria da situação da população brasileira.

Se tratando da política externa, além da busca pelo desenvolvimento nacional, havia também a busca pela autonomia internacional, sendo assim essa política se caracteriza por ser altiva e ativa, isto é, obstinada e com atitude para alcançar seus objetivos. Uma das formas para se chegar as duas condições eram a integração regional, como o acordo de mais 
tratados bilaterais e multilaterais e, portanto, a maior participação do Brasil no meio internacional, para assim se tornar um ator importante e decisivo.

A diplomacia cultural é uma ferramenta de construção da imagem de um país, também de um ator. No Governo Lula a diplomacia cultural tinha como objetivo construir a imagem de um Brasil pacifico, aberto ao diálogo e interessado em resolver conflitos de forma a evitar o uso da força. Avança uma estratégia de prevalência da negociação sobre o uso da violência para solução de conflitos e manutenção da paz (CERVO,2012, p. 540). Como dito antes, para se alcançar os objetivos da política externa, o Brasil usou da inserção internacional, principalmente na América do Sul, e aqui nesse ponto em que a diplomacia cultural tomou seu papel, através de instituições que o país participava - que serão melhores discutidas no próximo capítulo - o instrumento diplomático flui pelas vias de aproximação cultural, da propagação de intercâmbios, da solução de problemas em conjunto e etc.

O Presidente Luiz Inácio Lula da Silva e o Ministro das Relações Exteriores, Celso Amorim, buscaram caracterizar o Brasil como um mediador de conflitos no sistema internacional, assim cada vez mais ascendendo como um ator importante e decisivo. A diplomacia cultural empregada nesse meio entra na parte da construção no imaginário coletivo internacional da imagem do Brasil que o presidente e o ministro desejaram. Isto é, o propósito da diplomacia cultural brasileira se formulou para colocar o Brasil em três aspectos no meio internacional e assim produzir seu maior prestígio.

O primeiro aspecto é o de um Brasil pacífico, por optar as vias mais dialogadas, a diplomacia cultural usou dessa opção para passar a imagem de um país amigável, compreensivo e de soluções brandas. O segundo aspecto é o de Brasil mediador de conflitos, no governo Lula o país estabeleceu laços com a América do Sul, África, Oriente Médio e Ásia, lógico que com muitos propósitos, mas um deles, a busca por soluções sem intervir na política interna de seus parceiros, transformou o Brasil em um "famoso" mediador de conflitos, pois transparecia não querer tomar vantagem nas soluções que propunha. E por terceiro, o aspecto de um Brasil empático, como citado antes, o país fez laços novos e reascendeu antigos em várias partes do globo, e nesse contexto todo, o Governo Lula se interessava por assuntos de seus parceiros, mesmo que não de suma importância ao Brasil, isto é, os laços também se constituíram em quanto uma relação amigável, em que o país estava disposto a oferecer ajuda e suporte aos outros Estados.

Estes três aspectos fazem com que o Brasil ascenda no sistema internacional, assim assumindo uma posição mais influente, e seja prestigiado por outros atores, por sua amizade e capacidade empática.

Nestes três aspectos entram muitos elementos da cultura brasileira, que constituem a parte ideológica da construção da imagem do país, além das ações físicas. Manifestações culturais como Carnaval, tradições gastronômicas, a receptividade do povo brasileiro quanto aos estrangeiros, músicas, folclore popular, etc., também são parte da aparência do Brasil e possui influência no desenvolvimento dos aspectos citados. Essa influência é sentida no tocante a questões como insegurança e interesses em comum. 
$\mathrm{Na}$ América do Sul se partilha um fator em comum na formação dos Estados nacionais - a presença da colonização, já com o Oriente Médio, o Brasil se mostra receptivo quanto a seus indivíduos por ter um grande contingente populacional árabe no país. Situações assim favorecem relações mais próximas entre os atores.

\subsection{ESTRATÉGIAS E MEIOS}

Neste capítulo pretende-se explicitar as maneiras como, no Governo Lula, a diplomacia cultural foi usada para atingir os objetivos da política externa brasileira nesse período, que no caso é construir a imagem do Brasil como um país mediador de conflitos, optante pela via pacífica do diálogo.

Primeiramente sobre as estratégias usadas, pode-se dizer que, durante o governo em questão, fez parte do plano de política externa uma pluralização de alternativas estratégicas, ou seja, várias ações sendo realizadas concomitantemente. Diante desse portfólio, verificase a maior integração regional e firmação de acordos bi e multilaterais, isto é, o Brasil buscando, através da estratégia de inserção internacional, se tornar um ator com papel decisivo e de equilíbrio no sistema internacional. A estratégia tem por fim fortalecer laços com o maior número possível de nações, blocos e regiões, independentemente da posição geográfica (CERVO,2012, p. 533).

Segundamente, tratando dos meios usados, nota-se que o Brasil consegue se destacar em suas diversas facetas ao mesmo tempo, a cultura popular (literatura, arte, tradições) e nos diálogos políticos, isso quer dizer que, os meios utilizados tem como base o uso da cultura e da política.

$\mathrm{Na}$ questão da cultura popular, podemos verificar o Brasil se inserindo no sistema internacional através de eventos, em conjunto com outros países, em que promove seus costumes, danças, artes, buscando, também, no outro ator uma ideia em comum. Na prática tem como exemplo a maior participação do Brasil na Comunidade dos Países de Língua Portuguesa (CPLP), discurso do Ministro Celso Amorim, em 2007, no Dia da África, o I Festival de Música e Dança do IBAS (Índia, Brasil e África do Sul) em 2007, sediado em Salvador. Esses tipos de eventos dão a oportunidade dos países conhecerem novas tradições, mas também de encontrar interesses em comum, além de que, eventos culturais consolidam a identidade nacional de cada ator e também evita estereótipos negativos.

Já na questão política, temos exemplos a decisão conjunta entre Brasil e Honduras de um acordo que prevê a não obrigação de visto para indivíduos com passaporte simples válidos, de ambos os países, realizado em 2004, outro acordo firmado foi entre Croácia e Brasil em 2005, prevendo a isenção parcial de vistos para portadores de passaporte comum. Por fim, o Acordo de Cooperação Esportiva entre Governo da República Federativa do Brasil e o Governo do Estado de Catar. Aqui, o governo Lula usou da política para abrir caminhos para o intercâmbio cultural, e com uma agenda cheia de viagens e reunião, mostra como a política externa tem um papel importante no país, o que gera um certo prestígio para o Brasil.

A rede se tece também do lado da África e dos países árabes. Lula visitou a África uma dezena de vezes, além de provocar a cúpula países africanos-América Latina 


\begin{abstract}
e ser convidado de honra da Cúpula da União Africana. Programas na área de saúde, especialmente no combate à Aids, linhas de crédito, presença de empreiteiras brasileiras, atuação da Petrobras, integração com o Mercosul, exportações multiplicadas e voz comum contra os subsídios agrícolas contam entre os bons resultados alcançados por esta aproximação. Com a Comunidade dos Países de Língua Portuguesa (CPLP), se o retorno econômico e estratégico é baixo, o cultural é alto. A África vem criando condições favoráveis para atração de presenças estrangeiras, entre as quais a norte-americana, a chinesa e a brasileira se destacam. (CERVO, 2012, p. 556).
\end{abstract}

Nos eventos culturais não só há cultura popular, há também presença de almoços entre representantes dos Estados, celebrações em cúpulas, debates e diálogos em organizações regionais, como no Mercado Comum do Sul (MERCOSUL), entre outros, ou seja, nesses eventos o Brasil tem oportunidade de apresentar, não somente sua cultura, mas sua visão da política, econômica e social, assim pode, no olhar dos outros atores, ser um parceiro mais previsível, ou de interesses em comum.

Em síntese, os meios e estratégias usados no Governo Lula, incluem a diversificação de alternativas, que incluem a inserção internacional, integração regional e maior firmação de parceira com mais países, que foi realizada através da presença intensa do Brasil no sistema internacional, com acordos de cooperação envolvendo desde lazer a política, tratados, debates regionais e globais, celebrações de datas comemorativas em conjunto com outros países e eventos oficiais do Ministério de Relações Exteriores.

\title{
CONSIDERAÇÕES FINAIS
}

A diplomacia cultural sempre foi um instrumento muito vantajoso a diplomacia brasileira, já que antes mesmo de ser oficialmente posta em prática o Brasil já tinha estereótipos amigáveis atrelados a si, como a hospitalidade, a festança, a simpatia, entre outras características boas. Mas poucas vezes na história desse país a diplomacia cultural foi tão usufruída como no período do governo de Luiz Inácio Lula da Silva.

No período em questão, pode-se afirmar então que a diplomacia cultural foi usada com o intuito de inserir o Brasil no sistema internacional, de forma que esse assumisse a imagem de mediador - pacífico - de conflitos, isso é, um país que busca solucionar problemáticas considerando todos os lados e que não busca somente seus interesses mesquinhamente. Para chegar a esse propósito, o governo Lula utilizou de estratégias como a integração regional, buscando firmar acordos bilaterais e multilaterais com outros países fora de sua órbita tradicional, usufruindo das novas alternativas possibilitadas pelas novas parcerias, o que tem, também, como consequência a maior presença do Brasil no âmbito internacional, o caracterizando como um país influente.

Como um grande aprendizado dos teóricos de ciências humanas sabe-se que a teoria e prática tem de andar juntas para "surtir efeito". Com isso, na diplomacia cultural brasileira exercida no período em questão, nota-se que ao mesmo tempo, que pelo trabalho intenso dos diplomatas brasileiros e também do presidente, foi construído no imaginário internacional a ideia de um Brasil mediador de conflitos, simultaneamente, na prática, a agenda brasileira 
de eventos relacionados a política externa estava sempre cheia, que significa que o Brasil, além de dar importância a política externa, mostra seu empenho em se fazer presente, de estar presente, de sempre estar em dia com seus parceiros, em seus assuntos e também em suas resoluções. Ou seja, a diplomacia cultural no Governo Lula atuou muito bem na teoria e na prática, cuidando para que suas ações e falas fossem coerentes.

Outro ponto importante, digno de reflexão, é que se a cultura, usada como instrumento da diplomacia cultural, em um país tão vasto como o Brasil, consegue abranger a realidade cultural brasileira. Com tantas tradições diferentes, tantas maneiras de fazer arte, de literaturas nacionais regionais, músicas e danças, se torna complexo o funcionamento da diplomacia cultural no país em questão. Sabe-se que há dois estados brasileiros que são privilegiados no contexto internacional, e tem o privilégio de parecer a "cara" do Brasil, no caso são Rio de Janeiro e São Paulo, percebendo-se assim que as vezes algumas partes da realidade cultural brasileira são ignoradas. Importante saber, que isso ocorre não com tanta frequência, pois a agenda de política externa brasileira no período citado foi grande o bastante para abranger muitas regiões, mas que, a problemática de privilegiar algumas culturas regionais é sempre latente em uma país grande como o Brasil, digo, populoso e povoado.

\section{REFERÊNCIAS}

ALMEIDA, P. R. Uma Política Externa Engajada: a diplomacia do governo Lula. Revista Brasileira de Política Internacional v. 47, n. I, p. 162-184, 2004.

CARVAlHO, A. G. A Diplomacia Cultural Brasileira como Instrumento de Política Externa nos Governos Lula da Silva. II7 p. Trabalho de Conclusão de Curso Graduação em Relações Internacionais e Integração. Universidade Federal da Integração LatinoAmericana, Foz do Iguaçu, 2019.

CERVO, A. L.; BUENO, C. História da Política Exterior do Brasil Brasília: Instituto Brasileiro de Relações Internacionais. Editora da Universidade de Brasília, 2020.

MACHADO, G.L. A difusão cultural brasileira como instrumento de política externa: estratégias contemporâneas. Repositório Digital UFRGS, 2012. Disponível em: 〈https://www.lume.ufrgs.br/handle/ror83/71683>. Acesso em: 03 de set. de 2020.

MAPA, D. M. Inserção Internacional no Governo Lula: interpretações divergentes Revista Política Hoje, v. 19, n. I, 2010.

NYE, J. S. Soft Power: The Means to Success in World Politics. New York: Public Affairs, 2004.

RIBEIRO, E.T. Diplomacia Cultural: seu papel na política externa brasileira. Brasília: Fundação Alexandre de Gusmão, 2orı. 